\title{
A New Approach for Solving Weight Functions of Electromagnetic Flowmeters Using Resistive Network Modeling
}

\author{
Shiyi Yin and Bin Li \\ School of Mechatronics Engineering and Automation, Shanghai University, Shanghai 200072, China \\ Correspondence should be addressed to Shiyi Yin; ysy27@sina.com
}

Received 22 February 2013; Accepted 17 June 2013

Academic Editor: Oluwole Daniel Makinde

Copyright ( 92013 S. Yin and B. Li. This is an open access article distributed under the Creative Commons Attribution License, which permits unrestricted use, distribution, and reproduction in any medium, provided the original work is properly cited.

\begin{abstract}
The contribution of the flow signal is generally addressed by the weight function in the researches of the electromagnetic flowmeters, and various mathematical technologies were concentrated on the methodologies for solving the value of the weight function. However, it is still difficult to avoid the abstruse mathematical theories and the complex calculation when the solution domain is irregular in shape. This paper treats the problem within the intuitive physical perspective, and the approach, in which the proportion of the current is considered as the substitute for the weight function with the hypothetic current excitation source, is presented. A simple mathematical modeling of the current is built by means of the resistive network without the redundant assumption, and the strict mathematical derivation for the conventional asymmetric flow in the circular flowmeter is made to verify the feasibility and the correctness of the approach. The distributions of the weight function in various situations are obtained with the simulation employed, using the resistive network modeling, and the advantages of the approach are discussed.
\end{abstract}

\section{Introduction}

The study on the weight function is regarded as the general and essential focus in the field of the electromagnetic flowmeter (EMF). In 1954, the first analytical study for the EMF was performed by Shercliff [1] based on the twodimensional rectilinear flow and the uniformly distributed magnetic field. The microscopic characteristics of the induction electromotive force were demonstrated with the weight function method he proposed which is now widely used in analyzing the EMF. In 1970, Bevir [2] made further progress on the weight function theory with the virtual current introduced and extended it to the three-dimensional case. In 1971, Smyth [3] investigated the weight functions for the circular and rectangular channel EMFs by means of Green's function and conformal mapping. In 1983, O'Sullivan and Wyatt [4] researched the weight functions for electrodes with different geometries, and various maps of the distribution were obtained. In 1986, Hemp and Versteeg [5] solved the virtual current potential and the magnetic scalar potential by the expansion of Double Fourier series and simplified the calculation of the weight function significantly. In 1989, Zhang [6] proposed the alternating iteration method for solving the Laplace equation and applied it to achieve the semianalytical solution of the weight function for the EMF, on condition that the mixed boundary exists. In 2009, Hu et al. [7] divided the complex geometry by different auxiliary surfaces to obtain the corresponding analytical solutions of the weight function with the method of separation of variables.

Much effort has been made to solve the weight function for different situations by virtue of the mathematical skills in previous studies, based on the theory of the virtual current. The distribution of the weight function is especially the same as that of the virtual current if the magnetic field is uniform. However, the physical meaning of the weight function was less mentioned for the purpose of the calculation, by which the simple and direct approach to solve the weight function can be obtained.

In this paper, the theoretical background is briefly introduced, and the physical meaning of the weight function is proposed with the innovative modeling of the resistive network built, which results in the analytical solution of the weight function for the conventional asymmetric flow in the circular flowmeter. Besides, the simulations of the weight 
function are also involved to verify the correctness of the methodology addressed, and the conclusion is presented.

\section{Theoretical Background}

As is known, the basic measurement principle of the EMF is Faraday's Law, and the potential between two electrodes fulfills the integral equation which can be expressed as follows:

$$
U_{A B}=U_{A}-U_{B}=\int_{\tau} \vec{W} \cdot \vec{v} d \tau
$$

where $\vec{W}$ is given by

$$
\vec{W}=\vec{B} \times \vec{j}
$$

Here, $\vec{W}$ is the weight vector which is the extension of the weight function termed by Bevir and is considered as the general index for the distribution. $\vec{B}$ is the magnetic flux vector, and $\vec{j}$ is the current vector, which characterizes the current density on condition that the unit current passes from the positive electrode to the negative electrode. However, the current does not exist in the flow channel with the practical application of the EMF, and thus the current is called "virtual current." With the uniform magnetic field assumed, the distribution of the weight function is equal to that of the virtual current, and its value can be calculated by the partial differential equations.

Generally, there are no electric power and magnetic source inside the sensor, and the electrical potential $\varphi$ and magnetic potential $F$ within the flow area satisfy the Laplace' equations,

$$
\begin{aligned}
\nabla^{2} \varphi & =0 \\
\nabla^{2} F & =0,
\end{aligned}
$$

and the virtual current density $\vec{j}$ and magnetic flux vector $\vec{B}$ can be further denoted by

$$
\begin{aligned}
\vec{j} & =-\nabla \varphi \\
\vec{B} & =-\nabla F .
\end{aligned}
$$

According to (2), (3), and (4), the weight function is obtained by the following equation:

$$
\vec{W}=\nabla F \times \nabla \varphi=\left|\begin{array}{ll}
\frac{\partial F}{\partial y} & \frac{\partial F}{\partial z} \\
\frac{\partial \varphi}{\partial y} & \frac{\partial \varphi}{\partial z}
\end{array}\right| \vec{X}_{e}+\left|\begin{array}{ll}
\frac{\partial F}{\partial z} & \frac{\partial F}{\partial x} \\
\frac{\partial \varphi}{\partial z} & \frac{\partial \varphi}{\partial x}
\end{array}\right| \vec{Y}_{e}+\left|\begin{array}{ll}
\frac{\partial F}{\partial x} & \frac{\partial F}{\partial y} \\
\frac{\partial \varphi}{\partial x} & \frac{\partial \varphi}{\partial y}
\end{array}\right| \vec{Z}_{e}
$$

where $\vec{X}_{e}, \overrightarrow{Y_{e}}$, and $\vec{Z}_{e}$ are the unit vectors, respectively, in cartesian coordinate system.

From the previous equations, the solution of the weight function is mainly determined by the distribution of the

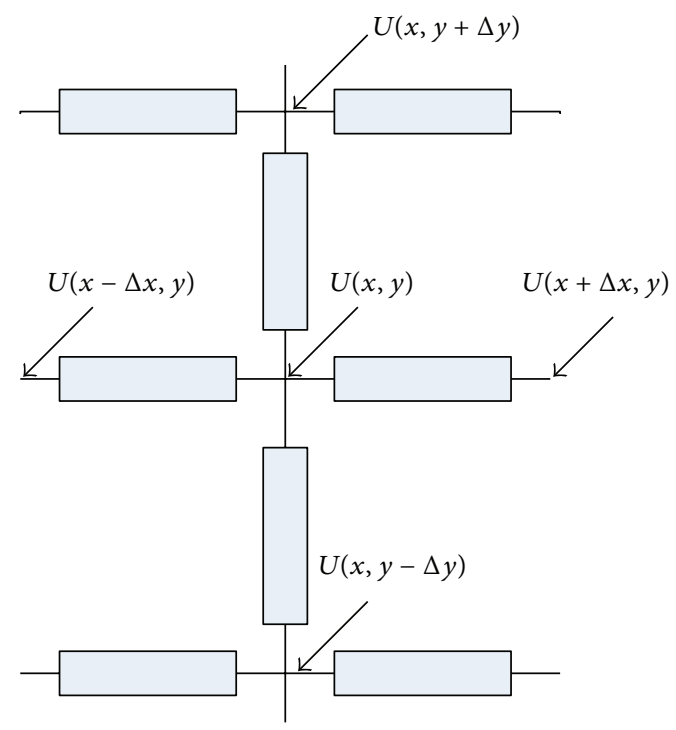

FIGURE 1: The cartesian grid of the resistive network.

virtual current which can be presented by the potential function. In previous researches, the methodologies for solving the direct analytical problems of the Laplace's equations to achieve the weight function were focused on, especially in the case of the various solution domains.

Different from the theory of the virtual current, this paper is concerned with the physical understanding of the weight function, which simply presents the proportion of the real current distribution. With the current excitation source assumed, the real current flows from the positive electrode to the negative electrode in the case of the static fluid, and the new modeling of the resistive network is established as the analogue of the flow, through which the weight function can be solved with an explicit physical explanation.

\section{Modeling and Derivation}

The EMF is generally assumed as the cylinder, and the electrodes are symmetrically placed in the circumference. The voltage signal is induced between the electrodes when the conductive fluid flows through the uniform magnetic field. The field theory analysis is made conventionally to build the virtual current model which depends on the mathematical skill of the partial differential equations other than the explicit physical meaning.

Based on the previous situation, a new approach for modeling is presented. The flow is regarded as the resistive network, and the current and potential in the fluid domain are solved with Kirchhoff's Current Law (KCL) employed.

Two different kinds of the grid system are considered in this model, the cartesian grid and the polar grid. The former grid shown in Figure 1 is established with several vertical and horizontal resistances. 


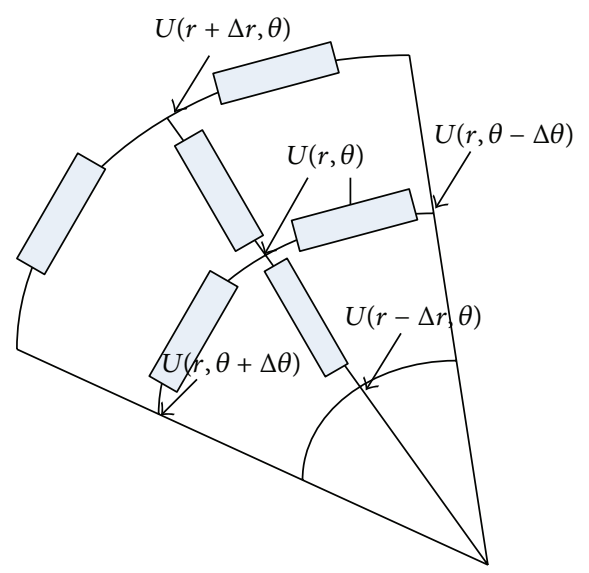

FIGURE 2: The polar grid of the resistive network.

According to the Kirchhoff's Current Law (KCL), in which the sum of the nodal current is zero, the mathematical expression is directly obtained as

$$
\begin{gathered}
\frac{U(x, y+\Delta y)-U(x, y)}{\rho \Delta y}+\frac{U(x, x+\Delta x)-U(x, y)}{\rho \Delta x} \\
+\frac{U(x, y-\Delta y)-U(x, y)}{\rho \Delta y} \\
+\frac{U(x, x-\Delta x)-U(x, y)}{\rho \Delta x}=0,
\end{gathered}
$$

where $\rho$ is the flow resistivity, and $\Delta x$ and $\Delta y$ are the unit step of the grid along the $x$ axis and the $y$ axis, respectively. Here, let $\Delta x=\Delta y=\Delta$, with the equal interval step introduced:

$$
\begin{gathered}
U(x, y+\Delta)+U(x, y-\Delta)+U(x+\Delta, y) \\
+U(x-\Delta, y)-4 U(x, y)=0 .
\end{gathered}
$$

The left part of the equation above is found to be the Laplacian operator, and (7) is equal to the equation $\nabla^{2} U(x, y)=0$, through which the feasibility and the correctness of the modeling using the resistive network can be verified. Nevertheless, it is difficult to approach the boundary with the cartesian grid utilized because of the pipe section in shape, and the latter grid, that is the polar grid, is then used to make up for the deficiency, demonstrated in Figure 2.

Similarly, the equation is established on the basis of the Kirchhoff's Current Law (KCL):

$$
\begin{gathered}
\frac{U(r+\Delta r, \theta)-U(r, \theta)}{\rho \Delta r}+\frac{U(r, \theta-\Delta \theta)-U(r, \theta)}{\rho(r \Delta \theta)} \\
+\frac{U(r-\Delta r, \theta)-U(r, \theta)}{\rho \Delta r} \\
+\frac{U(r, \theta+\Delta \theta)-U(r, \theta)}{\rho(r \Delta \theta)}=0 .
\end{gathered}
$$

The grid is generated minutely, such that

$$
\lim _{\substack{\Delta r \rightarrow 0 \\ \Delta \theta \rightarrow 0}} \frac{\Delta r}{r \Delta \theta}=1 .
$$

The partial differential equation is obtained with the combination of (8) and (9)

$$
\frac{\partial^{2} U}{\partial r^{2}}+\frac{1}{r^{2}} \cdot \frac{\partial^{2} U}{\partial \theta^{2}}=0 .
$$

With the method of separation of variables, the equation has the particular solution which has the form as follows:

$$
U(r, \theta)=R(r) \Phi(\theta)
$$

Substituting (11) into (10), the equation can be arranged as follows:

$$
\frac{r^{2}}{R} \frac{\partial^{2} R}{\partial r^{2}}=-\frac{1}{\Phi} \frac{\partial^{2} \Phi}{\partial \theta^{2}}=n^{2}
$$

where $n$ is the constant based on the structure of the equation.

Equation (12) is considered separately. The first section of it is the Euler equation, while the second section of it is the second-order linear homogeneous equation of constant coefficients. Then the analytical solution of the potential is

$$
\begin{aligned}
U(r, \theta) & =R(r) \Phi(\theta) \\
& =\sum_{n=0}^{\infty} C_{1} r^{\left(1+\sqrt{4 n^{2}+1}\right) / 2}\left[C_{3} \sin (n \theta)+C_{4} \cos (n \theta)\right] \\
& =\sum_{n=0}^{\infty} r^{\left(1+\sqrt{4 n^{2}+1}\right) / 2}\left[K_{1} \sin (n \theta)+K_{2} \cos (n \theta)\right]
\end{aligned}
$$

where $K_{1}$ and $K_{2}$ are denoted as the coefficient terms related the constant $n$.

The boundary condition is used to determine the coefficients. According to Kirchhoff's Current Law (KCL), the expression of the pipe boundary is obtained:

$$
\begin{aligned}
& \frac{U(r, \theta+\Delta \theta)-U(r, \theta)}{\rho(r \Delta \theta)} \\
& +\frac{U(r, \theta-\Delta \theta)-U(r, \theta)}{\rho(r \Delta \theta)} \\
& +\frac{U(r-\Delta r, \theta)-U(r, \theta)}{\rho \Delta r}=0 .
\end{aligned}
$$

The current represented by the first two terms of the equation does not exist because of the insulating material expect the electrodes in the senior, and the continuous form of (14) is

$$
\left.\frac{\partial U}{\partial r}\right|_{r=a}=0
$$

where $a$ presents the pipe radius. 
For the electrodes, it is generally regarded as the point charges, and the expression at the electrodes is conventionally set as a constant by means of the mathematical skill without the physical meaning. In this paper, with the assumed current excitation source, the expression at the electrodes can be obtained:

$$
\begin{gathered}
\left.\frac{\partial U}{\partial r}\right|_{r=a, \theta=\pi / 2}=\rho I, \\
\left.\frac{\partial U}{\partial r}\right|_{r=a, \theta=-(\pi / 2)}=-\rho I .
\end{gathered}
$$

Combined with (13), (15), and (16), the equation is arranged as follows:

$$
\begin{aligned}
& \sum_{n=0}^{\infty} \frac{1+\sqrt{4 n^{2}+1}}{2} a^{\left(\sqrt{4 n^{2}+1}-1\right) / 2}\left(K_{1} \sin n \theta+K_{2} \cos n \theta\right) \\
& = \begin{cases}\rho I & \theta=\frac{\pi}{2} \\
-\rho I & \theta=-\frac{\pi}{2} \\
0 & \forall \theta \neq \frac{\pi}{2},-\frac{\pi}{2} .\end{cases}
\end{aligned}
$$

Multiplying $\sin n \theta$ on the both sides of the equation, with the definite integration applied, (17) is arranged, and $K_{1}$ can be solved:

$$
\begin{aligned}
& \frac{1+\sqrt{4 n^{2}+1}}{2} \pi K_{1} \cdot a^{\left(\sqrt{4 n^{2}+1}-1\right) / 2}=2 \rho I \sin \frac{n \pi}{2} \\
& K_{1}=\frac{1}{1+\sqrt{4 n^{2}+1}} \cdot \frac{4 \rho I}{\pi} \sin \frac{n \pi}{2} a^{\left(1-\sqrt{4 n^{2}+1}\right) / 2} .
\end{aligned}
$$

It is noticed that $K_{1}$ is not equal to zero, so $n$ is the odd number. Assume that $n=2 m-1, K_{1}$ is transformed as

$$
K_{1}=\frac{(-1)^{m-1}}{1+\sqrt{4(2 m-1)^{2}+1}} \cdot \frac{4 \rho I}{\pi} a^{\left(1-\sqrt{\left.4(2 m-1)^{2}+1\right)} / 2\right.} .
$$

Similarly, multiplying $\cos n \theta$ on both sides of the equation, with the definite integration employed, (17) can also be arranged, and $K_{2}$ is determined:

$$
\begin{gathered}
\frac{1+\sqrt{4 n^{2}+1}}{2} \pi K_{2} \cdot a^{\left(\sqrt{4 n^{2}+1}-1\right) / 2}=0, \\
K_{2}=0 .
\end{gathered}
$$

By means of (13), (19), and (22), the analytical solution of the potential is

$$
\begin{aligned}
U(r, \theta)= & \sum_{m=1}^{\infty} r^{\left(1+\sqrt{4(2 m-1)^{2}+1}\right) / 2} \\
& \times\left\{\frac{(-1)^{m-1}}{1+\sqrt{4(2 m-1)^{2}+1}}\right. \\
& \left.\cdot \frac{4 \rho I}{\pi} a^{\left(1-\sqrt{\left.4(2 m)^{2}+1\right) / 2}\right.} \sin [(2 m-1) \theta]\right\} .
\end{aligned}
$$

For simplification, $\left(1+\sqrt{4(2 m-1)^{2}+1}\right) / 2$ is equivalent to $2 m-1$ in the infinite series, and then the potential is finally obtained as follows:

$U(r, \theta)$

$$
=\sum_{m=1}^{\infty} r^{2 m-1}\left\{\frac{(-1)^{m-1}}{2 m-1} \cdot \frac{4 \rho I}{\pi} a^{-(2 m-2)} \sin [(2 m-1) \theta]\right\} .
$$

Based on the Kirchhoff's Current Law (KCL), the radial current and the circumferential current of the arbitrary point in the flow domain are addressed as

$$
\begin{aligned}
I_{r} & =\lim _{\Delta r \rightarrow 0} \frac{U(r+\Delta r, \theta)-U(r, \theta)}{\rho \Delta r}=\frac{1}{\rho} \cdot \frac{\partial U(r, \theta)}{\partial r} \\
& =\frac{4 I}{\pi} \sum_{m=1}^{\infty}\left\{(-1)^{m-1}\left(\frac{r}{a}\right)^{2 m-2} \sin [(2 m-1) \theta]\right\}, \\
I_{\theta} & =\lim _{\Delta \theta \rightarrow 0} \frac{U(r, \theta+\Delta \theta)-U(r, \theta)}{\rho r \Delta \theta}=\frac{1}{\rho r} \cdot \frac{\partial U(r, \theta)}{\partial \theta} \\
& =\frac{4 I}{\pi} \sum_{m=1}^{\infty}\left\{(-1)^{m-1}\left(\frac{r}{a}\right)^{2 m-2} \cos [(2 m-1) \theta]\right\} .
\end{aligned}
$$

The $y$ axis is selected as the unified direction of the current; thus, the actual current is given by the sum of the component along the $y$ axis, including the radial current and the circumferential current, shown as follows:

$$
\begin{aligned}
I(r, \theta) & =I_{y}=I_{r} \sin \theta+I_{\theta} \cos \theta \\
& =\frac{4 I}{\pi} \sum_{m=1}^{\infty}\left\{(-1)^{m-1}\left(\frac{r}{a}\right)^{2 m-2} \cos [(2 m-2) \theta]\right\} .
\end{aligned}
$$

According to the definition, the weight function not only reflects the degree of the contribution of the flow signal generated from the motion of the flow through the magnetic field, but also describes the attenuation coefficient of the induced voltage caused by the geometric position of the points in the effective domain. From the physical meaning, the weight function is actually the distribution proportion. 


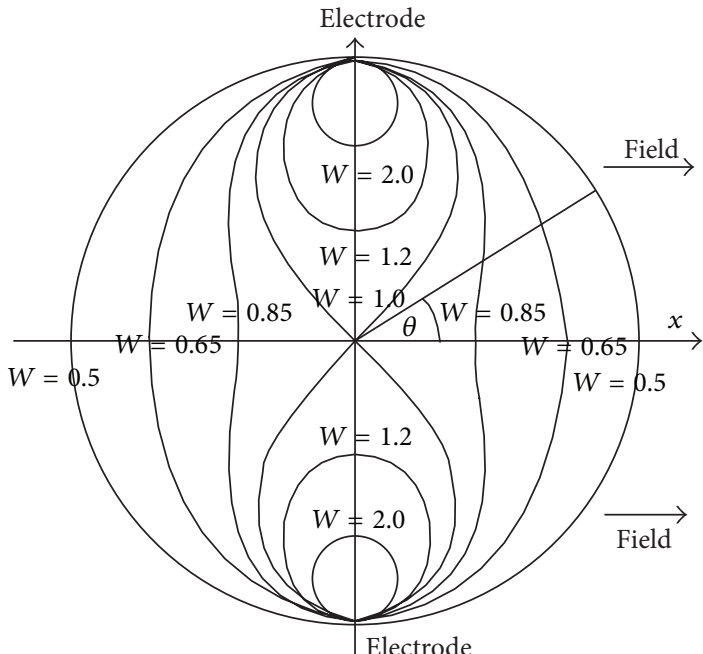

(a)

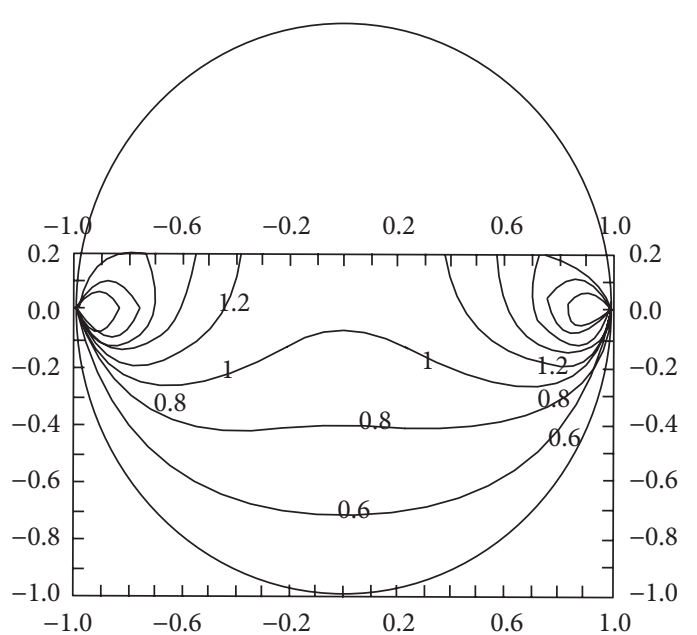

(c)

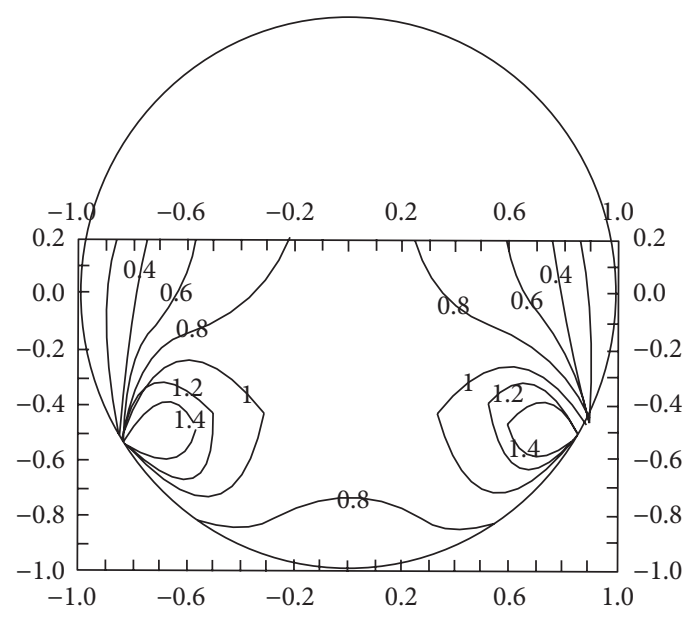

(e)

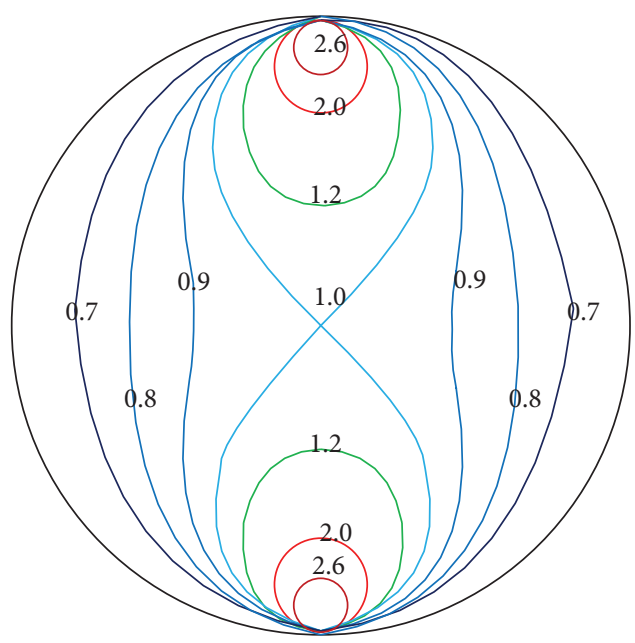

(b)

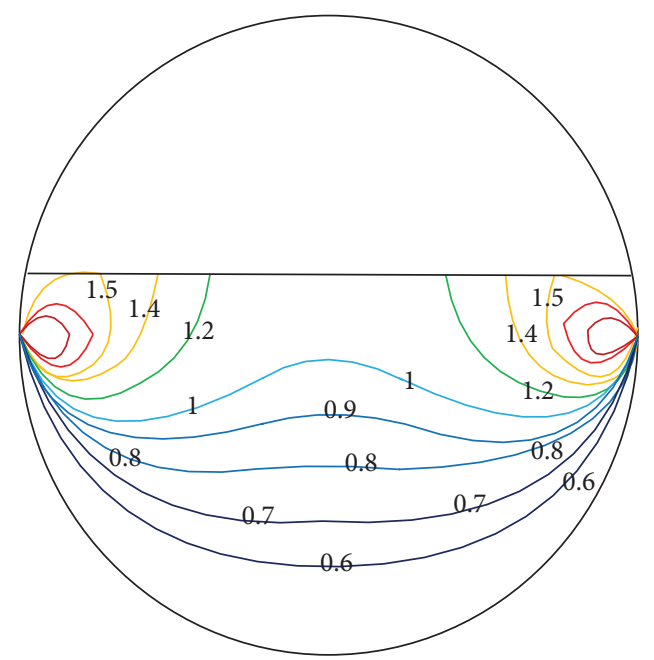

(d)

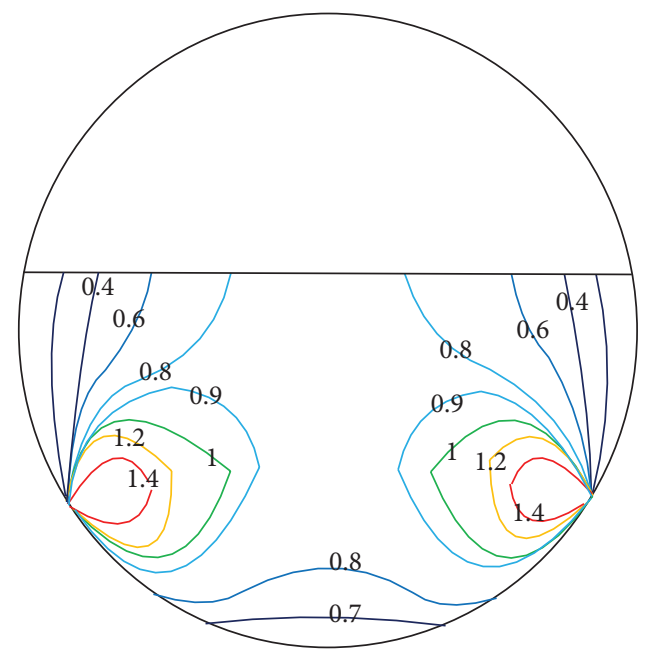

(f)

FIgURE 3: (a)-(f) The distributions of the weight function $[8,9]$ and the current simulated using the proposed approach. 


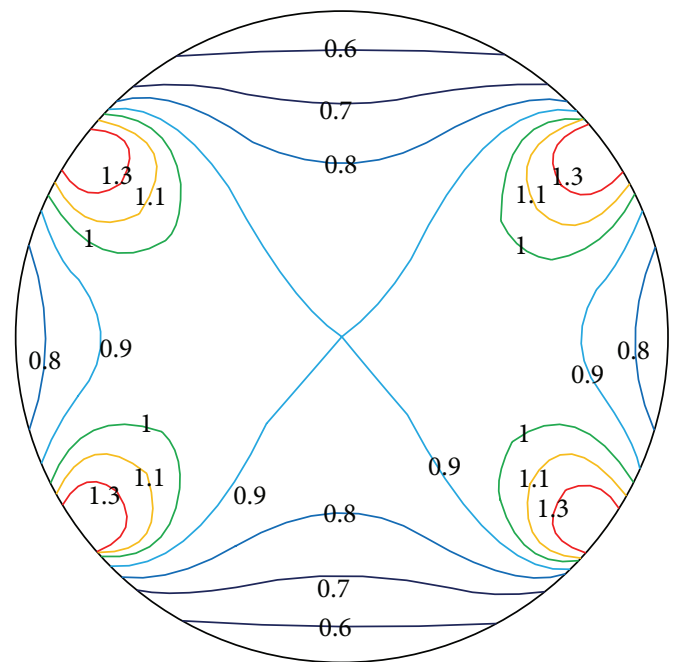

(a)

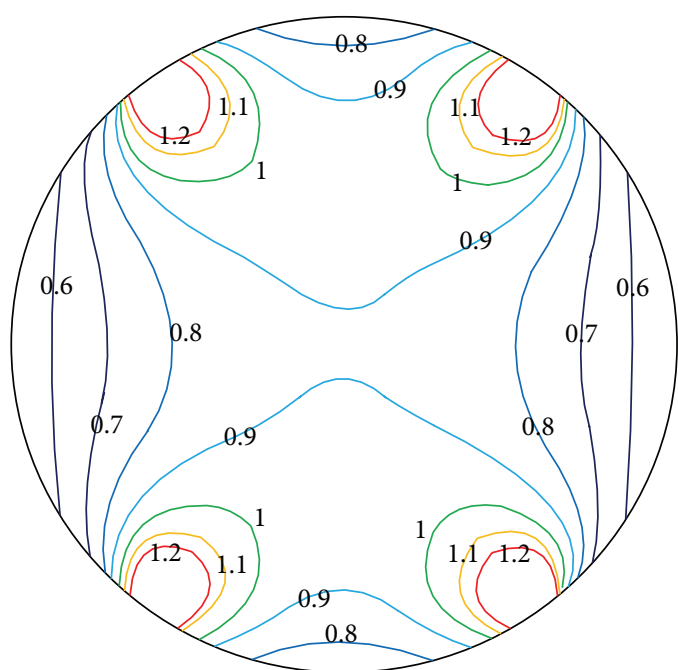

(b)

FIGURE 4: (a)-(b) The solutions of the weight function for EMFs with long-arc electrodes using resistive network modeling.

Thus, it is extended in this paper and regarded as the proportion of the current in the total current. Consider the following:

$$
\begin{aligned}
\widehat{W}(r, \theta) & =\frac{I(r, \theta)}{I} \\
& =\frac{4}{\pi} \sum_{m=1}^{\infty}\left\{(-1)^{m-1}\left(\frac{r}{a}\right)^{2 m-2} \cos [(2 m-2) \theta]\right\} .
\end{aligned}
$$

Let $N=m-1$; then (27) is arranged as

$$
\widehat{W}(r, \theta)=\frac{4}{\pi} \sum_{N=0}^{\infty}(-1)^{N} \cdot\left(\frac{r}{a}\right)^{2 N} \cos [(2 N) \theta] .
$$

On basis of the identities,

$$
\begin{aligned}
\sum_{N=0}^{\infty}(-1)^{N} \cdot\left(\frac{r}{a}\right)^{2 N} \cos [(2 N) \theta] & \\
= & \frac{1+\left(r^{2} / a^{2}\right) \cos 2 \theta}{1+\left(2 r^{2} / a^{2}\right) \cos 2 \theta+\left(r^{4} / a^{4}\right)} \\
= & \frac{a^{2}\left(a^{2}+x^{2}-y^{2}\right)}{a^{4}+2 a^{2}\left(x^{2}-y^{2}\right)+\left(x^{2}+y^{2}\right)^{2}},
\end{aligned}
$$

the analytical expression of the weight function is solved as

$$
\begin{aligned}
\widehat{W}(r, \theta) & =\frac{4}{\pi} \sum_{N=0}^{\infty}(-1)^{N} \cdot\left(\frac{r}{a}\right)^{2 N} \cos [(2 N) \theta] \\
& =K \cdot \sum_{N=0}^{\infty}(-1)^{N} \cdot\left(\frac{r}{a}\right)^{2 N} \cos [(2 N) \theta],
\end{aligned}
$$

where $K$ is the constant.
The normalization processing of the weight function is then made by dividing $\widehat{W}(0,0)$, where

$$
\begin{aligned}
\widehat{W}(0,0) & =\left.\frac{4}{\pi} \sum_{N=0}^{\infty}(-1)^{N} \cdot\left(\frac{r}{a}\right)^{2 N}\right|_{r=0} \\
& =\left.\frac{4}{\pi} \cdot \frac{1}{1+(r / a)^{2}}\right|_{r=0}=\frac{4}{\pi}=K .
\end{aligned}
$$

Thus, the normalized expression of the weight function is finally obtained:

$$
\begin{aligned}
W(r, \theta) & =\frac{\widehat{W}(r, \theta)}{\widehat{W}(0,0)} \\
& =\sum_{N=0}^{\infty}(-1)^{N} \cdot\left(\frac{r}{a}\right)^{2 N} \cos [(2 N) \theta] \\
& =\frac{1+\left(r^{2} / a^{2}\right) \cos 2 \theta}{1+\left(2 r^{2} / a^{2}\right) \cos 2 \theta+\left(r^{4} / a^{4}\right)} .
\end{aligned}
$$

With the transformation to the cartesian coordinate system, the solution is

$$
W(x, y)=\frac{a^{2}\left(a^{2}+x^{2}-y^{2}\right)}{a^{4}+2 a^{2}\left(x^{2}-y^{2}\right)+\left(x^{2}+y^{2}\right)^{2}} .
$$

\section{Results and Discussion}

Depending on (33), the solution of the weight function is exactly the same as the classical one, using the approach of the resistive network modeling, which validates the correctness of the scheme in this paper. From the simulation of the current with the modeling employed, it is demonstrated that the proportion of the current in the total current greatly reflects the weight function and it can be the analysis object 
for the research on the weight function with its modeling presented. In Figures 3(a) to 3(f), the weight functions for both full pipe and partially filled pipe are numerically identical to the proportion of the current in the total current from the simulation results.

In the previous studies on the classical situation, it was generally assumed that the electrodes were two point charges with different polarities, which caused the Neumann boundary condition, and the assumption of the conventional modeling previous brought the complexity to establish the Neumann equation in which the value of the partial derivative component determined by some mathematical skills [1, 6] is not explicit. With the characterization of the weight function by using the proportion of the current introduced in this paper, the mathematical constraint of the natural boundary condition is weakened, and the convenience for the calculation is provided.

With the methodology addressed, the weight functions for the EMFs which possess various kinds of electrodes can also be solved and the problems of the boundary condition are transformed to the difficulties of the modeling of the electrodes contacting the fluid, and thus, the reasonable selection of the model for boundary electrodes is essential by using the resistive network in the research on the weight function in the field of the electromagnetic flowmeter (see Figure 4).

\section{Conclusions}

The approach for solving the weight function is developed with the strict derivation of the mathematical expressions. The principle of the methodology is simple and effective, with the intuitive physical meaning of the weight function introduced, and it also possesses practical value to some extent in the field of the analysis on EMF. However, the boundary condition is various for different kinds of EMF, and the uniform modeling of the electrodes with complex geometric structure is to be further studied with this approach proposed.

\section{Acknowledgments}

The authors are grateful to the financial support of the Specialized Research Fund for the Doctoral Program of Higher Education of China (no. 20123108110011), titled "Research on the Weight Function and the Measurement of the EMF in Partially Filled Pipes".

\section{References}

[1] J. A. Shercliff, The Theory of Electromagnetic Flow-Measurement, Cambridge university press, London, UK, 1962.

[2] M. K. Bevir, "The theory of induced voltage electromagnetic flowmeter," Journal of Fluid Mechanics, vol. 43, pp. 577-590, 1970.

[3] C. C. Smyth, "Derivation of weight functions for the circular and rectangular channel magnetic flowmeters, by means of Green's theorem and conformal mapping," Journal of Physics E, vol. 4, no. 1, pp. 29-34, 1971.
[4] V. T. O'Sullivan and D. G. Wyatt, "Computation of electromagnetic flowmeter characteristics from magnetic field data. III. Rectilinear weight functions," Journal of Physics D, vol. 16, no. 8, pp. 1461-1476, 1983.

[5] J. Hemp and H. K. Versteeg, "Prediction of electromagnetic flowmeter characteristics," Journal of Physics D, vol. 19, no. 8, pp. 1459-1476, 1986.

[6] Z. X. Zhang, "A method for solving Laplace's equation with mixed boundary condition in electro magnetic flow meters," Journal of Physics D, vol. 20, pp. 573-576, 1989.

[7] L. Hu, J. Zou, X. Fu, H. Y. Yang, X. D. Ruan, and C. Y. Wang, "Divisionally analytical solutions of Laplace's equations for dry calibration of electromagnetic velocity probes," Applied Mathematical Modelling, vol. 33, no. 7, pp. 3130-3150, 2009.

[8] J. A. Shercliff, "Relation between the velocity profile and the sensitivity of electromagnetic flowmeters," Journal of Applied Physics, vol. 25, no. 6, pp. 817-818, 1954.

[9] X.-Z. Zhang, "The virtual current of an electromagnetic flow meter in partially filled pipes," Measurement Science and Technology, vol. 9, no. 11, pp. 1852-1855, 1998. 


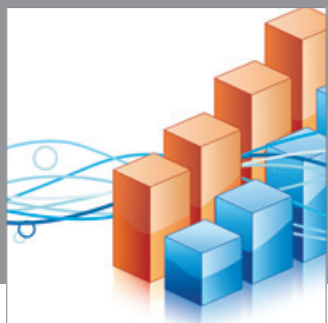

Advances in

Operations Research

mansans

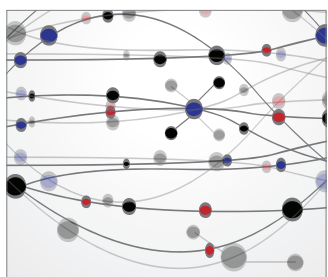

The Scientific World Journal
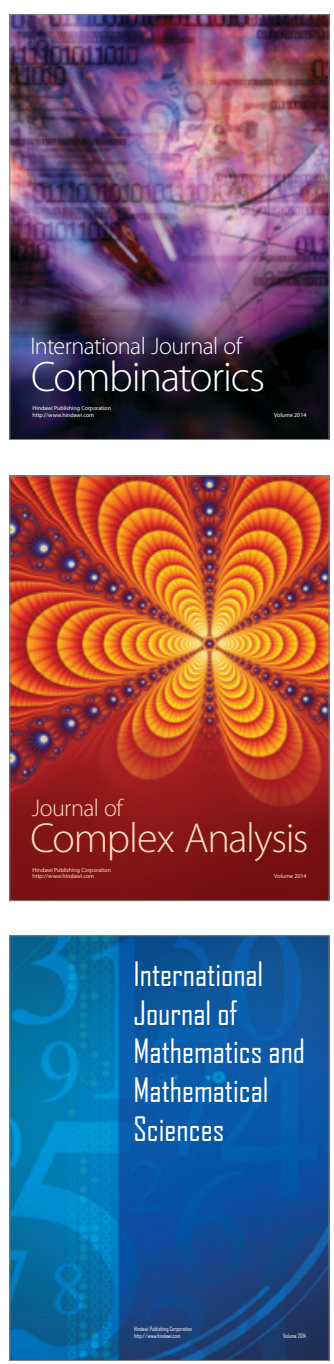
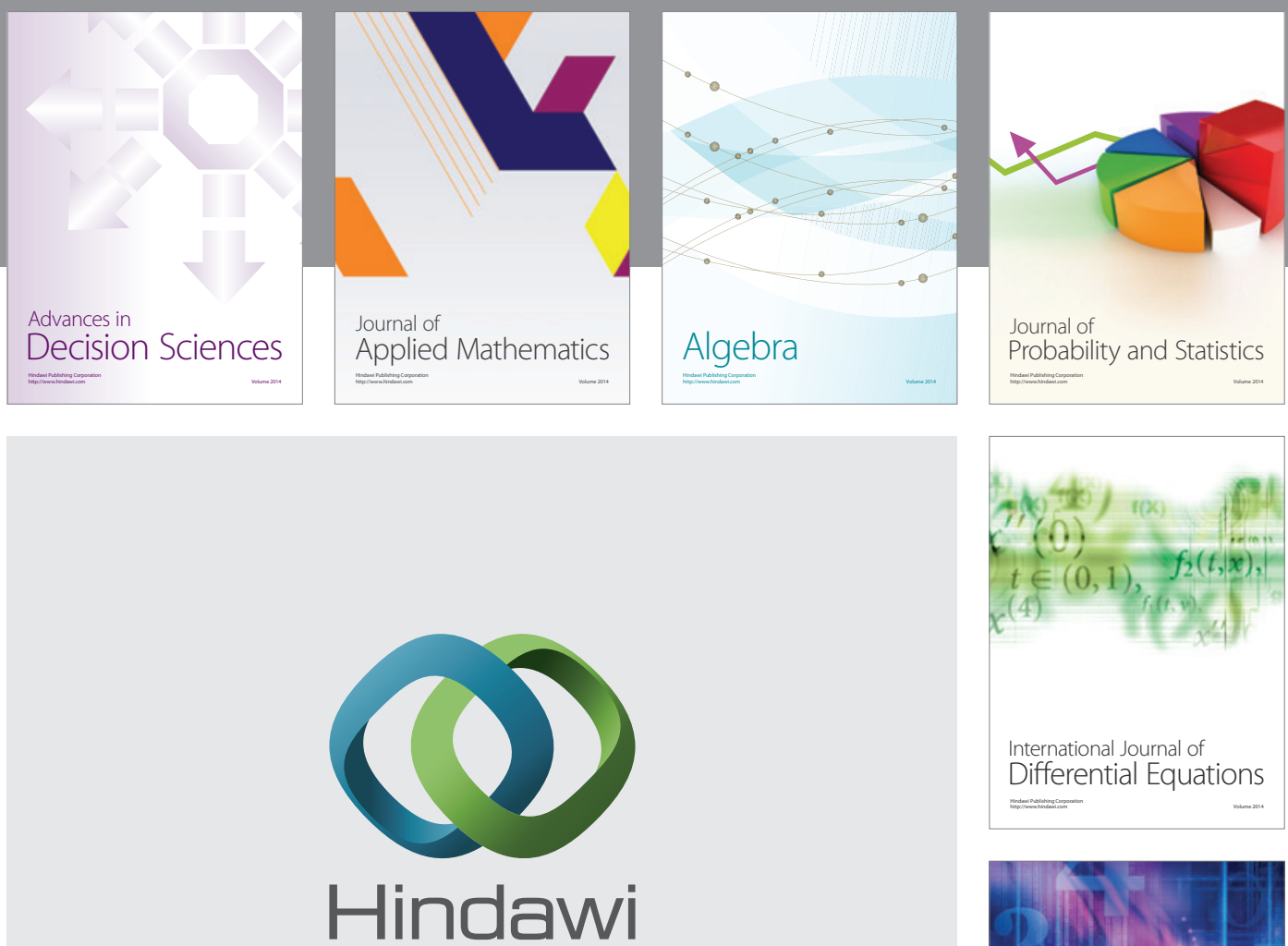

Submit your manuscripts at http://www.hindawi.com
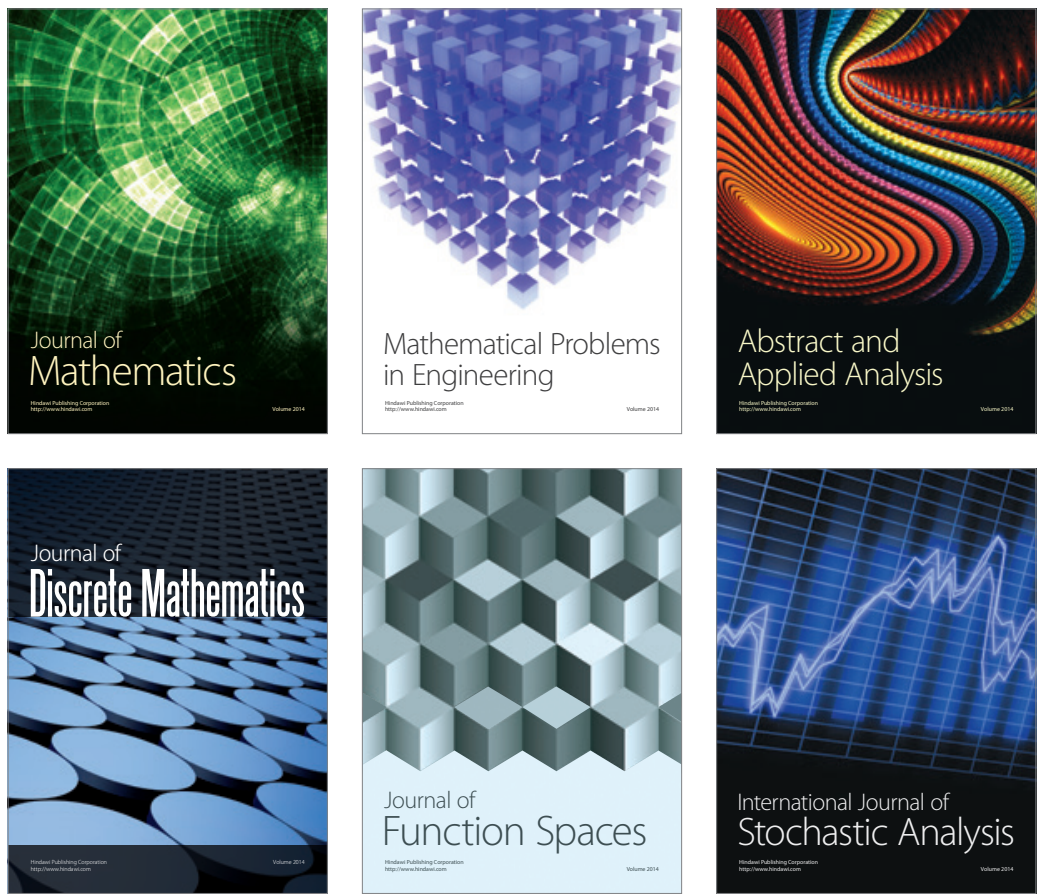

Journal of

Function Spaces

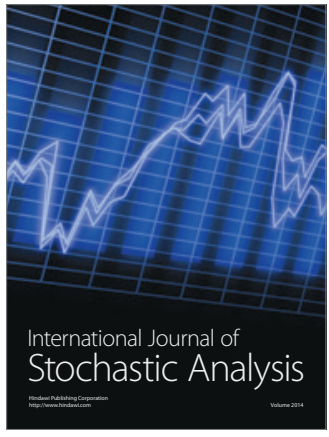

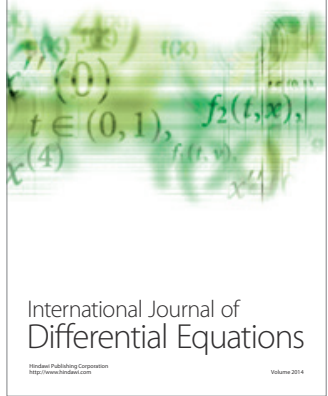
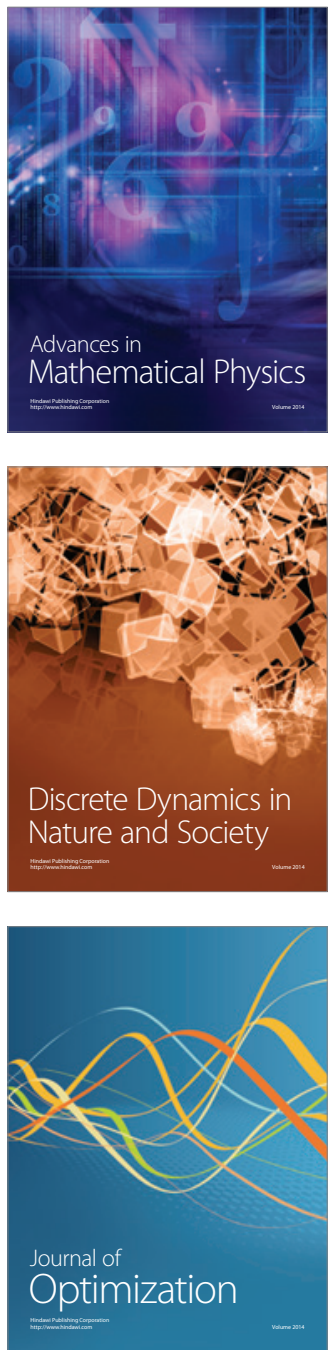